\title{
Respiratory muscle specific warm-up and elite swimming performance
}

\author{
Emma E Wilson ${ }^{1}$, Tricia M McKeever ${ }^{2}$, Claire Lobb ${ }^{3}$, Tom Sherriff ${ }^{4}$, Luke Gupta ${ }^{4}$, Glenn \\ Hearson ${ }^{1}$, Neil Martin ${ }^{5}$, Martin R Lindley ${ }^{4}$, Dominick E Shaw ${ }^{1}$
}

1. Nottingham Respiratory Research Unit (NRRU), School of Medicine, The University of Nottingham, UK

Clinical Sciences Building, Nottingham City Hospital, Hucknall Road, Nottingham, NG5 1PB

2. Division of Epidemiology and Public Health, School of Medicine, The University of Nottingham, UK

Clinical Sciences Building, Nottingham City Hospital, Hucknall Road, Nottingham, NG5 1PB

3. British Swimming, Loughborough University Swimming, Loughborough, UK Loughborough University, Epinal Way, Loughborough, Leicestershire, LE11 3TU

4. Department of Sports, Exercise and Health Science, Loughborough University, Loughborough, UK

Loughborough University, Epinal Way, Loughborough, Leicestershire, LE11 3TU

5. Institute for Lung Health, Glenfield Hospital, Leicester, UK

Glenfield Hospital, Groby Road, Leicester, LE3 9QP

\section{Correspondence:}

Dominick Shaw

Nottingham Respiratory Research Unit

Clinical Sciences Building

Nottingham City Hospital

Hucknall Road,

Nottingham,

NG5 1PB

Tel: 01158231709

Fax: 01158402617

Email: dominic.shaw@nottingham.ac.uk

\section{Keywords:}

Swimming, Respiratory, Inspiratory Muscle Training, Powerbreathe ${ }^{\circledR}$ 
Abstract:

\section{Background:}

Inspiratory muscle training has been shown to improve performance in elite swimmers, when used as part of routine training, but its use as a respiratory warm-up has yet to be investigated.

Aim:

To determine the influence of inspiratory muscle exercise (IME) as a respiratory muscle warm-up in a randomised controlled cross-over trial.

\section{Methods:}

A total of 15 elite swimmers were assigned to four different warm-up protocols and the effects of IME on 100m freestyle swimming times were assessed.

Each swimmer completed four different IME warm-up protocols across four separate study visits: swimming only warm-up; swimming warm-up plus IME warm-up (2 sets of 30 breaths with a $40 \%$ maximum inspiratory mouth pressure load using the Powerbreathe ${ }^{\circledast}$ inspiratory muscle trainer); swimming warm-up plus sham IME warm-up (2 sets of 30 breaths with a 15\% maximum inspiratory mouth pressure load using the Powerbreathe ${ }^{\circledR}$ inspiratory muscle trainer); and IME only warm-up. Swimmers performed a series of physiological tests and scales of perception (rate of perceived exertion and dyspnoea) at three time-points (pre warm-up, post warm-up and post time-trial).

\section{Results:}

The combined standard swimming warm-up and IME warm-up was the fastest of the four protocols with a $100 \mathrm{~m}$ time of 57.05 seconds. This was significantly faster than the IME only warm-up (Mean difference $=1.18 \mathrm{~s}, 95 \% \mathrm{Cl}: 0.44$ to $1.92, p<0.01$ ) and the swim only warm-up (Mean difference $=$ $0.62 \mathrm{~s}, 95 \% \mathrm{Cl}: 0.001$ to $1.23, p=0.05)$.

\section{Conclusion:}

Using IME combined with a standard swimming warm-up significantly improves $100 \mathrm{~m}$ freestyle swimming performance in elite swimmers. 


\section{Introduction:}

The goal of elite swimmers is to improve race performance and race times to achieve competitive success. The differences between winning and losing are small. Training has traditionally focused upon building skeletal muscle strength and endurance, more recently the focus has recently shifted to training the inspiratory musculature (1-4).

The untrained pulmonary system was originally considered able to cope with the additional stresses imposed by exercise (5), however several studies have demonstrated that training the inspiratory muscle system using an inspiratory muscle training regime (IMT) has additional benefits. IMT has been shown to enhance exercise performance in untrained (6-8) and trained individuals in several endurance sports (9-13) as well as during repeated sprinting $(14,15)$.

Competitive swimming presents several unique challenges to the respiratory system. Tight regulation of the breathing pattern (due to breath holding), at high flow rates and lung volumes, is required. Swim training has been shown to improve inspiratory muscle function (3) suggesting that swimming does place extra load on inspiratory dynamics (16-18).

The effect of IMT in competitive swimmers has been assessed previously. One study found that supplemental respiratory muscle training with 12 weeks of concurrent inspiratory and expiratory muscle training improved dynamic pulmonary function variables including forced inspiratory volume in one second $\left(F I V_{1}\right)$ and forced expiratory volume in one second $\left(F E V_{1}\right)$. There was no additional improvement above that of swim training alone on ventilatory response to hypercapnia, pulmonary function, sustainable breathing power or swimming performance. The study concluded that swimming training itself may well act as a form of IMT (4).

A more recent study from Kilding's group assessed the role of 6 weeks of IMT in a sham controlled trial of 16 competitive club-level swimmers. The IMT consisted of 30 repetitions, twice per day using a hand-held pressure threshold device. The IMT group had a small improvement in swim times over $100 \mathrm{~m}$ and $200 \mathrm{~m}$ with larger effects for maximal inspiratory pressure and rates of perceived exertion when compared to the sham control group (2).

Although a set period of IMT training in conjunction with normal swim training has been assessed, the effect of an IME specific warm-up has not. A warm-up is routinely employed by swimmers pre-race. There are few studies demonstrating the effects of a whole body warm-up on performance, but it is believed that warm-up may improve athletic performance (19-21) via a variety of biomechanical, physiological and biochemical responses (20-23), as well as accelerate the oxygen consumption response to subsequent exercise $(24,25)$. Prior exercise (in the form of a warm-up) may change the metabolic profile of exercise by speeding up overall oxygen uptake and blunting the blood lactate response (24). A warm-up is also intended to reduce the risk of injury (26). 


\section{Objective:}

We set out to examine whether incorporating an IME warm-up would improve $100 \mathrm{~m}$ freestyle swimming performance, pulmonary function and systemic markers of exercise induced stress in elite swimmers.

\section{Design:}

A total of 17 participants were initially contacted however two participants were not able to commit to the four sessions. A total of 15 participants from Loughborough University's elite swim team were recruited into the randomised single blind cross-over study with the help of British Swimming and Loughborough University. Elite swimmers were defined as those who were selected to represent British Swimming in international competitions. The athletes' swim specialities were butterfly $(n=3)$, breaststroke $(n=2)$, backstroke $(n=3)$ and freestyle $(n=7)$. There were a total of 9 male swimmers and 6 female swimmers, each swimmer completed all four warm-up protocols acting as their own controls (see Table 1).Each swimmer was randomly allocated to one of four different warm-up protocols each week for a period of four consecutive weeks. The sessions were all held at 9:00am on Tuesday mornings. All procedures were approved by the Loughborough University ethics committee and all participants consented to the requirements of the study in writing.

The primary outcome was $100 \mathrm{~m}$ freestyle sprint time-trial time following warm-up in a $25 \mathrm{~m}$ (shortcourse) pool.

Secondary outcomes included the following physiological parameters which were, measured both before and after the warm-up protocols, and before and after the $100 \mathrm{~m}$ freestyle time-trial:

1. Exhaled nitric oxide at $50 \mathrm{ml} / \mathrm{sec}$ (Aerocrine $\mathrm{NIOX} \mathrm{MINO}^{\circledR}$ technique, Aerocrine, Sweden.)

2. Spirometry Forced expiratory volume in 1 second $\left(F E V_{1}\right), F E V_{1}$ percent predicted, Forced Vital Capacity (FVC), FVC percent predicted, $\mathrm{FEV}_{1} / \mathrm{FVC}$ ratio, ratio percent predicted, Peak Flow (PEF), PEF percent predicted (using the Micromed spirometer, Micro Medical Ltd).

3. Maximum inspiratory and expiratory pressure $\left(\mathrm{cmH}_{2} \mathrm{O}\right)$ (using the MicroRPM (Respiratory Pressure Meter, Micro Medical Ltd)).

4. Ear lobe capillary blood lactate ( $\mathrm{mmol} / \mathrm{L})$ (Lactate Pro LT-1710, Fact Canada Consulting Ltd).

5. Heart rate (bpm) and arterial blood oxygen saturation $\left(\mathrm{PaO}_{2}\right)$ (using Pulse Oximeter, Micro Medical Ltd).

6. Rate of perceived exertion scale (27).

7. Dyspnoea scale (27).

The four warm-up protocols were:

\section{Protocol 1:}


Standard swimming warm-up consisting of $2500 \mathrm{~m}$ ( $100 \times 25 \mathrm{~m}$ lengths), with a mixture of all four strokes (butterfly, backstroke, breaststroke and freestyle), leg only work (kicking with a float), arm only work (using a pull buoy) and underwater work.

\section{Protocol 2:}

A respiratory muscle specific warm-up, using the Powerbreathe ${ }^{\circledast}$ inspiratory muscle trainer. A standard protocol was followed consisting of two sets of 30 inspirations at $40 \%$ of maximal inspiratory muscle pressure. $40 \%$ load was used to prevent undue fatigue during the warm-up. See below for determination inspiratory pressures.

\section{Protocol 3:}

Standard swimming warm-up (protocol 1) was performed followed by sham IME warm-up with a resistance load equal to $15 \%$ maximal inspiratory muscle pressure.

\section{Protocol 4:}

Standard swimming warm-up (protocol 1) followed by the IME warm-up (protocol 2).

\section{$\underline{\text { Inspiratory Muscle Pressure Settings }}$}

The swimmers had their Powerbreathe ${ }^{\circledR}$ device set up individually: Each participant performed maximum inspiratory muscle pressure manoeuvres using the MicroRPM (Respiratory Pressure Meter) until 5 measurements within $5 \%$ of each other were obtained. The maximum value of those 5 measurements was set as the maximum inspiratory muscle pressure for each participant.

None of the swimmers were told which load they were using (sham of $15 \%$, active of $40 \%$ ) but were informed that different loads were being compared rather than an active versus sham effect. The Powerbreathe ${ }^{\circledR}$ device was set up with a resistance load equal to $40 \%$ maximum inspiratory muscle pressure for the IME only warm-up (protocol 2) and the combined warm-up (protocol 4). Each participant was assigned the same Powerbreathe ${ }^{\circledR}$ device for each session.

\section{Statistical Analysis}

The analysis was performed using repeated measures ANOVA to detect the $100 \mathrm{~m}$ freestyle time trial differences between the four different warm-up protocols. We checked for cofounders in age, $\mathrm{FEV}_{1}$ and predominant stroke, but we found nothing which indicated an adjustment was required. The same process was used to detect differences between pre warm-up, post warm-up and post time-trial measurements in physiological parameters. The differences were analysed between pre warm-up vs. post warm-up, pre warm-up vs. post time-trial and post warm-up vs. post time-trial. All statistical analyses were performed using STATA 11. 


\section{Results:}

The 15 participants completed $100 \%$ of the sessions and $100 \%$ of the physiological measurements.

Table 1: descriptive baseline demographics (pre-intervention) for the participants studied. Data displayed as percentage or (mean \pm standard deviation).

\begin{tabular}{|l|r|}
\hline \multicolumn{2}{|c|}{ Table 1: Demographics Table } \\
\hline Number of participants & 15 \\
\hline Gender: Male (\%) & $9(60 \%)$ \\
\hline Gender: Female (\%) & $6(40 \%)$ \\
\hline Age (Years) & $21.2 \pm 1.6$ \\
\hline Height (cm) & $180.17 \pm 7.84$ \\
\hline Weight (kg) & $75.2 \pm 9.05$ \\
\hline Baseline FEV 1 (L) & $4.84 \pm(0.81)$ \\
\hline Baseline FEV ${ }_{1} \%$ Predicted & $112 \pm(12.19)$ \\
\hline Baseline FVC (L) & $5.90 \pm(0.95)$ \\
\hline Baseline FVC \% Predicted & $115 \pm(9.14)$ \\
\hline Baseline Peak Flow (L/min) & $600 \pm(134)$ \\
\hline Baseline F $_{E} N O$ 50ml/sec (ppb) & $29 \pm(20)$ \\
\hline
\end{tabular}

$F E V_{1}=$ Forced Expiratory Volume in 1 second

$F V C=$ Forced Vital Capacity

$F_{E} N O=$ Fraction of Exhaled Nitric Oxide

There were significant differences between the four different warm-up protocols and the resulting $100 \mathrm{~m}$ freestyle times. The fastest protocol was swimming plus IME warm-up with a $100 \mathrm{~m}$ time of 57.05 seconds (See Table 2). The swimming plus IME warm-up was significantly faster than both the IME only warm-up (Mean difference $=1.18$ seconds, $95 \% \mathrm{Cl}: 0.44$ to $1.92, \mathrm{p}<0.01$ ) and the swimming only warm-up (Mean difference $=0.62$ seconds, $95 \% \mathrm{Cl}$ : 0.001 to $1.23, p=0.05$ ). The swimming plus IME warm-up was faster than the swimming plus sham IME warm-up, however the difference was not statistically significant (Mean difference $=0.33$ seconds, $95 \% \mathrm{Cl}:-0.44$ to $1.11, p=1.00$ ). The IME only warm-up was also significantly faster than the swimming plus sham IME warm-up (Mean difference = 0.85 seconds, $95 \% \mathrm{Cl}: 0.05$ to $1.65, \mathrm{p}=0.035)$.

There were no significant differences between any other of the warm-up protocols. Nor were there any differences in physiological parameters between the four different warm-up protocols across the groups. 
Table 2: Results for $100 \mathrm{~m}$ swimming performance.

\begin{tabular}{|l|l|l|l|l|l|}
\hline & $\begin{array}{l}\text { Swim and IME } \\
\text { warm-up }\end{array}$ & $\begin{array}{l}\text { Swim and sham } \\
\text { IME warm-up }\end{array}$ & $\begin{array}{l}\text { Swimming only } \\
\text { warm-up }\end{array}$ & $\begin{array}{l}\text { IME only } \\
\text { warm-up }\end{array}$ & $\begin{array}{l}\text { ANOVA } \\
p^{-v a l u e *}\end{array}$ \\
\hline 100 m time (s) & 57.05 & 57.39 & 57.67 & 58.24 & 0.0001 \\
\hline
\end{tabular}

* ANOVA shows a statistically significant difference between the four groups.

\section{Discussion:}

The use of a swimming warm-up utilising IME appeared to have significant benefits to performance. The use of a combined swimming plus IME (with the Powerbreathe ${ }^{\circledR}$ device) warm-up improved $100 \mathrm{~m}$ swimming performance by 0.62 seconds when compared to a standard swimming warm-up alone and resulted in the fastest swimming time over $100 \mathrm{~m}$ (57.05 seconds). There were no significant differences observed in any of the physiological parameters measured across the four different warmup protocol groups. This was expected as the study was of short duration and there was no training effect over time, as the IME device was used as part of a warm-up protocol rather than for long-term training. Changes in swimming performance are measured in small time-trial and physiological increments, and many factors contribute to overall performance, the study controls as many factors as possible; including time of day, race lane and swim wear.

To our knowledge this is the first randomised controlled study of IME as an adjunct to swimming warm-up and performance. The sample size was consistent with another study (2) evaluating IME as an aid to swim training. Other strengths included each participant acting as their own control, measurements being performed at the same time of day and the use of a sham technique.

Our study suffers from weaknesses similar to others in this area; the numbers were relatively small, swim performance can vary by small amounts in an unpredictable manner, and we did not employ a group that had no warm-up at all. The fact that there was no significant difference between sham IME and swim versus swim only warm-up is reassuring and suggests that the sham IME was a true placebo.

Our study finding is similar to others (2). Kilding et al. (2010), used IME as a part of a training routine over a period of six weeks in 16 competitive club-level swimmers at $50 \%$ load. Their group found an improvement in $100 \mathrm{~m}$ and $200 \mathrm{~m}$ swimming performance, but not $400 \mathrm{~m}$. The study demonstrated a significant improvement in the rate of perceived exertion in swimmers who used IME. The study highlighted the complex nature of the mechanisms underlying changes in inspiratory muscle function and improvements in performance.

Precise mechanistic explanations for the observed improvement in performance seen in this study and others have been proposed. These include an increase in the threshold for activation of the 
inspiratory muscle metaboreflex (28-30) and modification of fatigue perception via a central metabolic control/central (brain) governor (31). Other studies suggest that the mechanism for respiratory muscle specific warm-up is neural in origin $(16,32)$. It is possible that these effects occurred in our study too. Another explanation is that the use of IME results in an augmentation of blood flow to respiratory muscles. Given the use of IME as a warm-up with a relatively low load over a short time course it seems unlikely that warm-up IME results in long term physiological changes in the respiratory muscles. Consistent with this, there were no differences in physiological parameters between the four warm-up protocols.

\section{Conclusion:}

In conclusion we have shown that IME employed as a tool for warm-up improves swimming performance. The mechanisms behind these improvements require significant further investigation. Given the magnitude of change seen in performance the use of IME in a warm-up regime should be explored further.

\section{Conflict of interest:}

The authors declare they have no conflict of interest.

\section{Acknowledgements:}

\section{Funding Sources:}

IMT and other clinical test equipment were funding by:

Nottingham Respiratory Research Unit, Clinical Sciences Building, Nottingham City Hospital, Hucknall Road, Nottingham, NG5 1PB and Department of Sports, Exercise and Health Science, Loughborough University, Epinal Way, Loughborough, Leicestershire, LE11 3TU

\section{Thanks:}

Staff and coaches at Loughborough University Swimming Pool

British Swimming

Volunteers from Loughborough University

\section{Contributorship:}

EW, NM, ML, DS conceived and designed the study. EW, TS, LG, ML obtained approvals. EW, CL. TS, LG, GH, ML managed the study. EW, GH, TM, DS, ML analysed the data. All authors contributed to the interpretation of the data and revision of the final manuscript. EW and DS wrote the first draft of the manuscript. DS and ML are guarantors. 
Page 9 of $\mathbf{1 2}$ 


\section{Summary:}

What are the new findings?

- IME has not been used as a warm-up regime previously. Traditionally it has been used as a long-term (six weeks plus) training aid.

- IME as a warm-up device in addition to a standard swimming warm-up can improve swimming performance in elite swimmers.

- No physiological or biochemical parameters were shown to change with the use of IME warmup.

How might it impact upon clinical practice in the near future?

- Further investigation is required into the use of IME as a warm-up device, but it is a practical, safe and simple addition to the standard swimming warm-up.

- Warming-up the respiratory muscles and the respiratory system in addition to the cardiovascular and muscular-skeletal system is likely to demonstrate added performance benefits given the results of the study.

- Elite swimming performance may be improved with the use of an IME warm-up device, which is beneficial for success at international competitions. 


\section{References:}

1. Jakovljevic DG, McConnell AK. Influence of different breathing frequencies on the severity of inspiratory muscle fatigue induced by high-intensity front crawl swimming. J Strength Cond Res. 2009 Jul;23(4):1169-74.

2. Kilding $A E$, Brown $S$, McConnell AK. Inspiratory muscle training improves 100 and 200 m swimming performance. Eur J Appl Physiol. 2010 Feb;108(3):505-11.

3. Mickleborough TD, Stager JM, Chatham K, Lindley MR, Ionescu AA. Pulmonary adaptations to swim and inspiratory muscle training. Eur J Appl Physiol. 2008 Aug; 103(6):635-46.

4. Wells GD, Plyley M, Thomas S, Goodman L, Duffin J. Effects of concurrent inspiratory and expiratory muscle training on respiratory and exercise performance in competitive swimmers. Eur J Appl Physiol. 2005 Aug;94(5-6):527-40.

5. Amann M, Hopkins WG, Marcora SM. Similar sensitivity of time to exhaustion and time-trial time to changes in endurance. Med Sci Sports Exerc. 2008 Mar;40(3):574-8.

6. Edwards AM, Cooke CB. Oxygen uptake kinetics and maximal aerobic power are unaffected by inspiratory muscle training in healthy subjects where time to exhaustion is extended. Eur J Appl Physiol. 2004 Oct;93(1-2):139-44.

7. Gething $A D$, Williams $M$, Davies $B$. Inspiratory resistive loading improves cycling capacity: a placebo controlled trial. Br J Sports Med. 2004 Dec;38(6):730-6.

8. Sheel AW. Respiratory muscle training in healthy individuals: physiological rationale and implications for exercise performance. Sports Med. 2002;32(9):567-81.

9. Edwards AM, Wells C, Butterly R. Concurrent inspiratory muscle and cardiovascular training differentially improves both perceptions of effort and $5000 \mathrm{~m}$ running performance compared with cardiovascular training alone. $\mathrm{Br}$ J Sports Med. 2008 Oct; $42(10): 823-7$.

10. Griffiths LA, McConnell AK. The influence of inspiratory and expiratory muscle training upon rowing performance. Eur J Appl Physiol. 2007 Mar;99(5):457-66.

11. Johnson MA, Sharpe GR, Brown PI. Inspiratory muscle training improves cycling time-trial performance and anaerobic work capacity but not critical power. Eur J Appl Physiol. 2007 Dec;101(6):761-70.

12. Romer LM, McConnell AK, Jones DA. Effects of inspiratory muscle training on time-trial performance in trained cyclists. J Sports Sci. 2002 Jul;20(7):547-62.

13. Volianitis S, McConnell AK, Koutedakis Y, McNaughton L, Backx K, Jones DA. Inspiratory muscle training improves rowing performance. Med Sci Sports Exerc. 2001 May;33(5):803-9.

14. Romer LM, McConnell AK, Jones DA. Effects of inspiratory muscle training upon recovery time during high intensity, repetitive sprint activity. Int J Sports Med. 2002 Jul;23(5):353-60.

15. Tong TK, Fu FH, Chung PK, Eston R, Lu K, Quach B, et al. The effect of inspiratory muscle training on high-intensity, intermittent running performance to exhaustion. Appl Physiol Nutr Metab. 2008 Aug;33(4):671-81.

16. Lomax M, Castle S. Inspiratory muscle fatigue significantly affects breathing frequency, stroke rate, and stroke length during 200-m front-crawl swimming. J Strength Cond Res. 2011 Oct;25(10):2691-5.

17. Lomax M, Iggleden C, Tourell A, Castle S, Honey J. Inspiratory muscle fatigue after race-paced swimming is not restricted to the front crawl stroke. J Strength Cond Res. 2012 Oct;26(10):2729-33.

18. Brown $S$, Kilding AE. Exercise-induced inspiratory muscle fatigue during swimming: the effect of race distance. J Strength Cond Res. 2011 May;25(5):1204-9.

19. Fradkin AJ, Zazryn TR, Smoliga JM. Effects of warming-up on physical performance: a systematic review with meta-analysis. J Strength Cond Res. 2010 Jan;24(1):140-8.

20. Hedrick A. Exercise Physiology: Physiological Responses to Warm-up. National Strength and Conditioning Association Journal. 1992;14(5):25-7. 
21. Shellock FG, Prentice WE. Warming-up and stretching for improved physical performance and prevention of sports-related injuries. Sports Med. 1985 JulAug;2(4):267-78.

22. Bishop D. Warm up II: performance changes following active warm up and how to structure the warm up. Sports Med. 2003;33(7):483-98.

23. Bishop D. Warm up I: potential mechanisms and the effects of passive warm up on exercise performance. Sports Med. 2003;33(6):439-54.

24. Burnley $M$, Doust $J H$, Jones AM. Effects of prior warm-up regime on severeintensity cycling performance. Med Sci Sports Exerc. 2005 May;37(5):838-45.

25. Hajoglou A, Foster C, De Koning JJ, Lucia A, Kernozek TW, Porcari JP. Effect of warm-up on cycle time trial performance. Med Sci Sports Exerc. 2005 Sep;37(9):160814.

26. Woods $\mathrm{K}$, Bishop $\mathrm{P}$, Jones $\mathrm{E}$. Warm-up and stretching in the prevention of muscular injury. Sports Med. 2007;37(12):1089-99.

27. Borg GA. Psychophysical bases of perceived exertion. Med Sci Sports Exerc. $1982 ; 14(5): 377-81$.

28. Chiappa GR, Roseguini BT, Vieira PJ, Alves CN, Tavares A, Winkelmann ER, et al. Inspiratory muscle training improves blood flow to resting and exercising limbs in patients with chronic heart failure. Journal of the American College of Cardiology. 2008 Apr 29;51(17):1663-71.

29. McConnell AK, Lomax $M$. The influence of inspiratory muscle work history and specific inspiratory muscle training upon human limb muscle fatigue. The Journal of physiology. 2006 Nov 15;577(Pt 1):445-57.

30. Witt JD, Guenette JA, Rupert JL, McKenzie DC, Sheel AW. Inspiratory muscle training attenuates the human respiratory muscle metaboreflex. The Journal of physiology. 2007 Nov 1;584(Pt 3):1019-28.

31. Edwards AM, Walker RE. Inspiratory muscle training and endurance: a central metabolic control perspective. Int J Sports Physiol Perform. 2009 Mar;4(1):122-8.

32. Hawkes EZ, Nowicky AV, McConnell AK. Diaphragm and intercostal surface EMG and muscle performance after acute inspiratory muscle loading. Respir Physiol Neurobiol. 2007 Mar 15;155(3):213-9. 